infection. Effective partner notification (PN) is key to identifying sexual contacts that may have been exposed, providing the opportunity to offer necessary advice, support and testing.

Aim This audit explored current PN practices across the North East in order to identify opportunities for improvement and inform future guidance.

Methods All genitourinary medicine and infectious diseases clinics across the North East were asked to complete questionnaires for up to ten newly diagnosed cases of HIV between January and December 2010, and provide additional background information on PN arrangements. Data were analysed used EpiData version 3.1.

Results Seven out of nine (78\%) clinics responded. PN was discussed with $82 \%(46 / 56)$ of newly diagnosed patients and documented in the records of $77 \%$ (43/56). The time taken to complete all PN outcomes ranged from 0-29 weeks (median 1.5 weeks) and a mixture of methods were used to calculate the PN period. A total of 70 regular partners were recorded, 32 of which were tested and $44 \%$ (14/32) found to be HIV positive. 95 casual partners were recorded, nine of which were tested and none found to the HIV positive. Additional outcomes such as safe sex discussions and condom use were generally less frequently documented.

Discussion and Conclusion Although this sample is relatively small, there was engagement from the majority of regional services, suggesting that it is likely to be representative of local practice. It is clear that there is variation in current PN practices between clinics, and there are a number of challenges which may be particularly pertinent to HIV infection. These findings will be used to inform local policy and standards with the aim of improving the quality of local services and ensuring accountability for actions

\section{P30 AN ANALYSIS OF PATIENT CHARACTERISTICS ASSOCIATED WITH GONOCOCCAL RESISTANCE TO PAST THERAPEUTIC AGENTS IN ENGLAND AND WALES}

doi:10.1136/sextrans-2012-050601c.30

${ }^{1} \mathrm{~K}$ Town, ${ }^{* 1} \mathrm{C} \mathrm{M}$ Lowndes, ${ }^{1} \mathrm{~S}$ Chisholm, ${ }^{1} \mathrm{M}$ Kall, ${ }^{1} \mathrm{E}$ Webster, ${ }^{1} \mathrm{~T}$ Nichols, ${ }^{1} \mathrm{~J}$ Anderson, ${ }^{1} \mathrm{C}$ Obi, ${ }^{2} \mathrm{~B}$ Willey, ${ }^{1} \mathrm{C}$ Ison. ${ }^{1}$ Health Protection Agency; ${ }^{2}$ London School of Hygiene and Tropical Medicine, London, UK

Background Antimicrobial resistance in Neisseria gonorrhoeae restricts effective treatment options. Recent UK treatment guidelines recommend ceftriaxone as first line treatment. If ceftriaxone becomes unsuitable for treating gonorrhoea there are no suitable available alternatives. The potential for using past therapeutic agents for gonorrhoea treatment should be assessed.

$\operatorname{Aim}(\mathbf{s}) / \mathbf{O b j e c t i v e ( s ) ~ I d e n t i f y ~ r i s k ~ f a c t o r s ~ a s s o c i a t e d ~ w i t h ~ a n t i - ~}$ microbial resistance to penicillin, tetracycline and ciprofloxacin.

Methods Data from the Gonococcal Resistance to Antimicrobials Surveillance Programme were analysed for trends in antimicrobial resistance across sub-groups of the Gonococcal Resistance to Antimicrobials Surveillance Programme population. Using 2010 data patient variables associated with penicillin, tetracycline and ciprofloxacin resistance were identified using univariate and multivariable analyses of ORS.

Results Rates of penicillin, tetracycline and ciprofloxacin resistance have increased across all regions in England and Wales in recent years, with very high levels found in white MSM. Lower rates of resistance, with $<15 \%$ prevalence, indicating a susceptible population, are found in women (penicillin $6.4 \%$, ciprofloxacin $12.8 \%$ ), black ethnicity (penicillin 6.1\%, ciprofloxacin 13.4\%), and in some cases heterosexual men (penicillin 13.2\%) and those aged under 24 (penicillin 12.6\%). Univariate and multivariable analysis identified patient variables, including sexual orientation, ethnicity and age as strongly associated with penicillin, tetracycline and ciprofloxacin resistance.
Conclusions By using sub-group population prevalence data on resistance, as well as information about patient variables strongly associated with resistance, it may be possible in some cases to adapt treatment regimens to make use of previously recommended antimicrobials and preserve the use of ceftriaxone for high-risk groups.

\section{P31 HOW IMPORTANT IS IT TO KNOW WHERE INDEX PATIENTS MEET THEIR SEXUAL PARTNERS IN ORDER TO CONTROL THE TRANSMISSION OF SEXUALLY TRANSMITTED INFECTIONS?}

doi:10.1136/sextrans-2012-050601c.31

R Ford, ${ }^{*}$ M Clarke, I Lloyd, N Speare, K Maleham, R Robertshaw, C Morgan. Bristol Sexual Health Centre, Bristol, UK

Background Learning where people have met their sexual partners has previously been acknowledged as a way of identifying key locations that are functional to sexually transmitted infection transmission.

Aims To gather local data on where patients diagnosed with chlamydia, gonorrhoea, syphilis, HIV, trichomonas and hepatitis B have met their partners. This is the first stage of an on-going project which aims to develop a more targeted health promotion intervention(s) for key locations in Bristol identified as being important for STI transmission. The ultimate aim is that this intervention(s) will contribute to a reduction in STI transmission rates and therefore STI prevalence(s) in Bristol.

Method Patients were asked where they met their sexual partners during all health adviser partner notification interviews conducted in 2011. 2052 interviews were conducted and data were collected from 1257 patients.

Results Across all patients, the most regularly cited meeting place was in a bar or club (29\%) This was followed by "through friends/ old friends" (28\%). 12\% met their sexual contacts via the internet. The most common mode of meeting contacts in heterosexual patients was via friends (31\%) while for gay men it was via the internet (36\%)

Discussion The data provides the names of key locations in Bristol and a number of internet sites that are important for transmission. These locations can now be targeted by health promotion and outreach work in order to contribute to a reduction in STI transmission and prevalence. Over a quarter of all sexual contacts were met via friends or they were a friend of the patient. It is likely that the perception of STI risk diminishes if a contact is known or if they share the same social network. As well as targeting locations and internet sites, further research is now needed to explore patient's perception of STI risk, alongside health promotion work that highlights potential misperceptions.

\section{P32 PARTNER NOTIFICATION (PN) WITHIN NON-SPECIALIST SERVICES}

doi:10.1136/sextrans-2012-050601c.32

C Cunningham, ${ }^{*} \mathrm{~K}$ McCormick. NHS Tayside, Dundee, UK

Background The local board has the highest rates of diagnosis of genital Chlamydia in Scotland. PN is critical to breaking the chain of infection but there is a clear lack of consistency in its provision outside the specialist services.

Aim To meet the NHS Health Improvement Scotland Standard 4.2 for Sexual health services; "Individuals are offered partner notification in all settings delivering sexual healthcare, including in primary care, youth services and community pharmacies". 